\title{
Prinzmetal's angina or/and ectatic atherosclerotic coronary artery disease?
}

\section{Tomislava Bodrožić \\ Džakić Poljak*, \\ (Piana Rudan, \\ Miroslav Raguž, \\ (-) Jasmina Ćatić}

University Hospital Dubrava, Zagreb, Croatia

\author{
KEYWORDS: vasospastic angina, functional coronary disease. \\ CITATION: Cardiol Croat. 2017;12(9-10):387-388. I https://doi.org/10.15836/ccar2017.387 \\ *ADDRESS FOR CORRESPONDENCE: Tomislava Bodrožić Džakić Poljak, Klinička bolnica Dubrava, Avenija Gojka \\ Šuška 6, HR-10000 Zagreb, Croatia. / Phone: +385-98-9691-881 / E-mail: tobodrozic@gmail.com \\ ORCID: Tomislava Bodrožić Džakić Poljak http://orcid.org/0000-0002-7293-3972 \\ Diana Rudan http://orcid.org/0000-0001-9473-2517 • Miroslav Raguž http://orcid.org/0000-0003-1567-8503 \\ Jasmina Ćatić http://orcid.org/0000-0001-6582-4201
}

IIIIIIIIIIIIIIIIIIIIIIIIIIIIIIIIIIIIIIIIIIIIIIIIIIIIIIIIIIIIIIIIIIIIIIIIIIIIIIIIIIIIIIIIIIIIIIIIIIIIIIIIIIIIIIIIIIIIIII

Introduction: Ischemic heart disease is entity that involves structural atherosclerotic coronary artery disease (SCAD) and functional disorder of coronary circulation (Figure 1). Functional disorder of coronary artery is vasospastic angina (known as Prinzmetal's, variant or inversa. This type of angina is rare, representing about $2 \%$ cases of angina. ${ }^{1}$ We present the very rare case of a male patient who has coronary artery disease that involves both; structural -ectatic and functional-occlusive coronary artery disease (CAD).

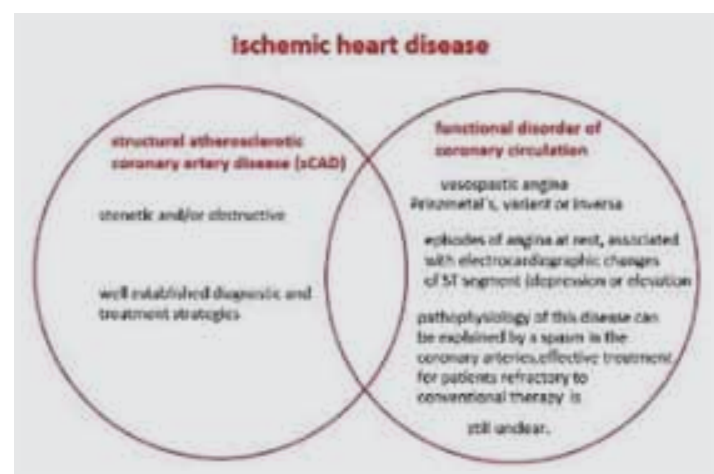

FIGURE 1. Schematic overview of coronary artery disease.
Case report: 61-old male patient, with history of smoking (90pack per year) and arterial hypertension presented in emergency department with high blood pressure (240/130mmHg), epistaxis and headache. He complained that he has been suffering from severe chest pain in early mornings and after exposure to cold air. The chest discomfort lasted for five minutes with spontaneous resolving and was not precipitated by exercise. The initial electrocardiogram (ECG) did not show any signs of ischemia and laboratory parameters, including troponin were in normal range. Due to history of repeating chest pain, he was admitted to the Department of Cardiology for further investigation. Echocardiography showed moderate, symmetric, concentric hypertrophic myocardial muscle, preserved left ventricular systolic function and no valvular abnormalities. Coronarography was also performed. After contrast administration in left coronary artery patient complained of chest pain; ECG showed ST segment elevation up to $2 \mathrm{~mm}$ in all precordial leads and the occlusion of medial left anterior descendens branch (LAD) due to spasm could be seen (Figure $\mathbf{2}$ and $\mathbf{3}$ ). After parenteral

RECEIVED:

September 24, 2017

ACCEPTED:

September 26, 2017

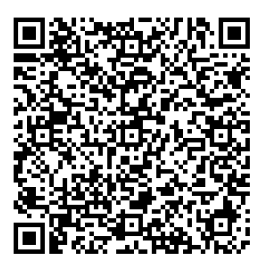

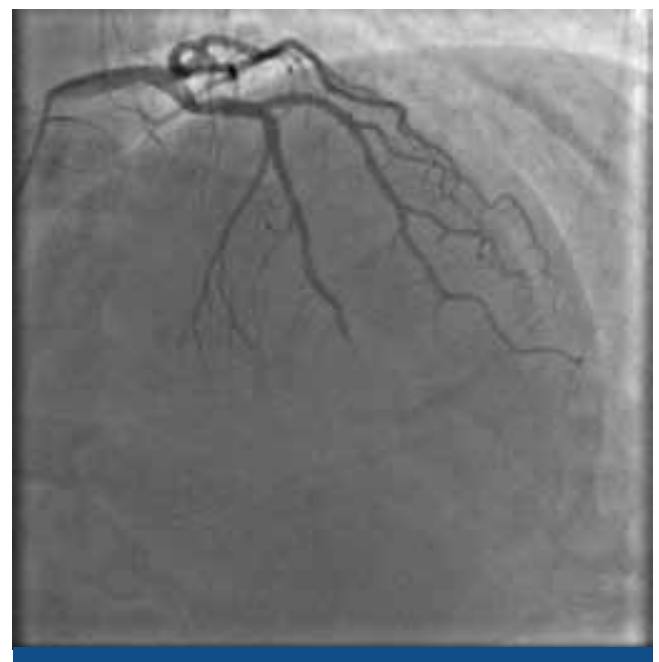

FIGURE 2. Occlusion of the medial segment of the left anterior descending branch during contrast application - right anterior obliquecranial view.

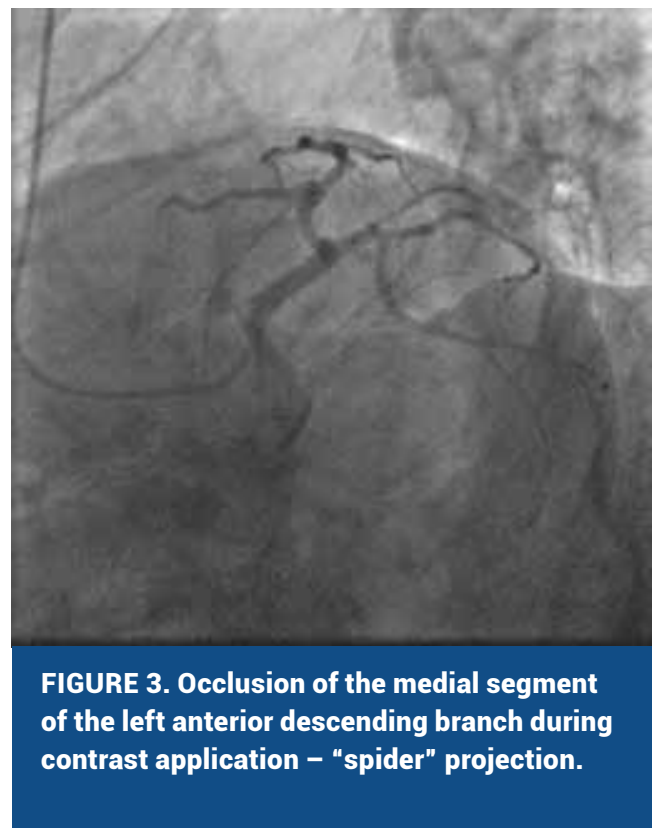


Prinzmetal's angina or/and ectatic atherosclerotic

coronary artery disease?

administration of nitrates the flow was established to the distal LAD (Figure 4). Right coronary angiography showed ectatic, dominant right coronary artery, with 50\% stenosis in proximal segment (Figure 5). After contrast application patient had chest pain and ST segment denivelation in inferior ECG leads with no evident significant stenosis. We suggested treatment with calcium-channel blockers, angiotensin converting enzyme inhibitor, acetylsalicylic acid and statin.

Conclusion: It can be concluded that our patient has both: the vasospastic angina and the atherosclerotic coronary artery disease. Sometimes, it is hard to decide what kind of further diagnostic and therapeutic approach should be taken because of reasonable doubt which lesion (like in our patient) is "culprit" one.
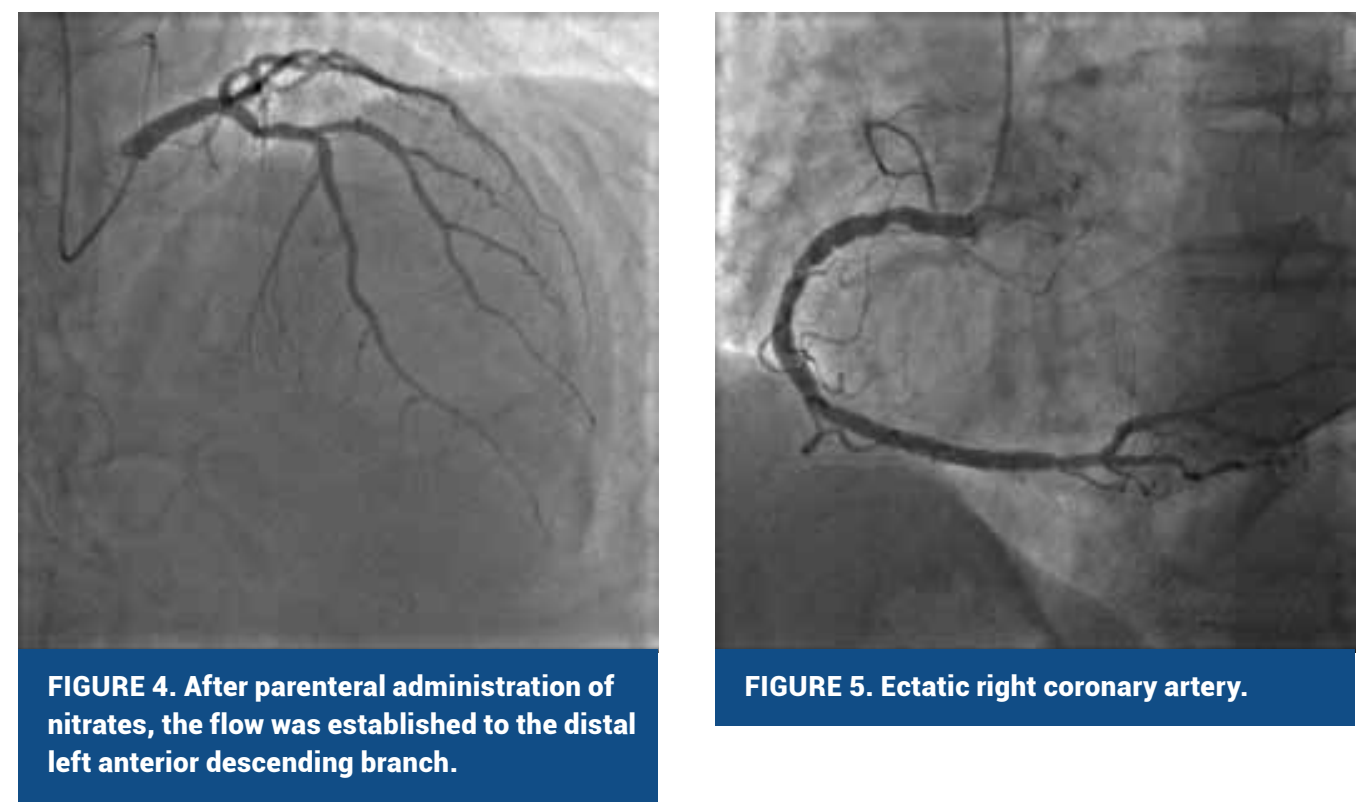

\section{LITERATURE IIIIIIIIIIIIIIIIIIIIIIIIIIIIIIIIIIIIIIIIIIIIIIIIIIIIIIIIIIIIIIIIIIIIIIIIIIIIIIIIIIIIIIIIIIIIIIIIIIIIIIIIIIIIIIIIIIIIIIIIIIIIIIIIIIIIII}

1. Beltrame JF, Crea F, Kaski JC, Ogawa H, Ong P, Sechtem U, et al; Coronary Vasomotion Disorders International Study Group (COVADIS). The Who, What, Why, When, How and Where of Vasospastic Angina. Circ J. 2016;80(2):289-98. https://doi.org/10.1253/circj.CJ-15-1202 